\section{Using hillslope-scale groundwater model to bridge the gap between basin inputs and river concentrations. The case of nitrates in Brittany (France).}

\author{
LUCA GUILLAUMOT ${ }^{1}$, LUC AQUILINA ${ }^{2}$, JEAN \\ MARÇAIS $^{3}$, PATRICK DURAND ${ }^{4}$ AND JEAN-RAYNALD \\ DE DREUZY ${ }^{5}$
}

${ }^{1}$ International Institute for Applied Systems Analysis

${ }^{2}$ Université Rennes 1

${ }^{3}$ INRAe

${ }^{4}$ INRAE

${ }^{5} \mathrm{CNRS}$

Presenting Author: luca.guillaumot@live.fr

Hydrogeological modelling has been intensively used to understand groundwater functioning and manage groundwater resources in the critical zone. It remains however difficult to link groundwater fluxes to river. Residence time, one of the parameters resulting from modelling, is difficult to estimate. Residence time is however particularly important to predict pollutant heritage for diffuse pollutions such as agriculture nitrate and to make the link between groundwater storage and fluxes and river nitrate concentrations.

We developed a new modelling strategy using a simple and parsimonious hillslope model with uniform hydrological parameters. These models can be straightforwardly deployed (19,000 modeling experiments) in order to investigate a wide range of situations. They include seepage areas which allow to reproduce rapid flow $(<1 \mathrm{yr})$ while groundwater provides both medium and long-term ranges of residence times (1-100 yrs). They have been applied to 3 catchments in Brittany-France an area of intensive agriculture.

The model is calibrated using river flow, long-term nitrate chronicles and CFC measurements in deep and shallow wells, springs and river. These tracers provide clear residence time and provide strong constraints on the groundwater volume.

The large range of models used allows to reproduce specific characteristics of catchments. It also allows to define residence times of the various components of groundwater (surface young water, shallow subsurface, deep subsurface) which contribute to the river. In addition, the typical groundwater stratification modeled gives strong insights about denitrification properties of catchments.

A global mass balance of the nitrate mass fate in the catchment is presented. 50 to $70 \%$ of nitrate applied during the considered period (1955-2010) has been flushed out by the river with a mean residence time of 8 to $12 \mathrm{yrs}$ while 16 to $40 \%$ is still stored in the aquifer with a mean residence time of 9 to 17 yrs. About 9 to $21 \%$ has been denitrified.

Finally, the model is used to precisely quantify the impact of nitrate application modifications in the river with time.

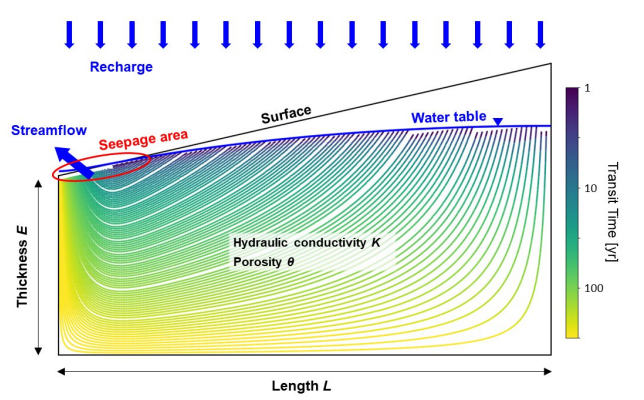

